\title{
Composite Materials Based on Ultra High Molecular Weight Polyethylene and Rare-earth Element Oxides
}

\author{
ALEXEY MIKHAILOVICH NEMERYUK, MARINA MIKHAILOVNA LYLINA, \\ MARINA VLADIMIROVNA BOGDANOVSKAYA, ELENA ALEKSANDROVNA AVERINA \\ and ANTON SERGEYEVICH YEGOROV*
}

\author{
The Federal State Unitary Enterprise «Institute of Chemical Reagents and High Purity Chemical \\ Substances of National Research Centre «Kurchatov Institute»107076, \\ Bogorodsky val, 3. Moscow, Russia. \\ *Corresponding author E-mail: egorov@ irea.org.ru \\ http://dx.doi.org/10.13005/ojc/340253
}

(Received: December 07, 2017; Accepted: February 10, 2018)

\begin{abstract}
A method of producing composite materials based on ultra high molecular weight polyethylene (UHMWPE) and metal oxides, such as rare-earth elements oxides (REE oxides), including gadolinium, dysprosium and tungsten oxides, was developed in this study. The surface of oxide fillers was modified using organosilicon coupling dressing agents in the acid catalysis environment. The modification allows to produce compositions containing significant amount of filler (over $30 \%$ ). The effect of introduction of rare earth oxides into the matrix of ultrahigh molecular polymer up to $40 \%$ by weight on thermal and oxidative degradation was studied.
\end{abstract}

Keywords: Ultra high molecular weight polyethylene, Polymer composite, Metal oxide, Rare earth elements.

\section{INTRODUCTION}

Composite materials based on carbo-chain polymers and inorganic fillers are widely used in various fields due to such characteristics as higher wear resistance and adverse environment resistance, including photodegradation and aggressive media effects $^{1-4}$.
Depending on the nature of a carbo-chain polymer, there are various approaches to producing filled composites. To produce compositions containing significant amount of filler (over $60 \%$ ), it is possible to use a method based on monomer polymerization over filler, with a polymer layer forming on the surface of filler particles, followed by threating the powder produced at higher 
temperatures and under pressure application to create solid blanks from the powder produced?

Specific ultra high molecular weight polyethylene (UHMWPE) properties impose a number of limitations on the applicability of widely used methods of producing highly-filled compositions. Thus, to produce UHMWPE-based materials that have improved physical and mechanical characteristics, it is possible to use a technology of impregnating UHMWPE powder with precursors, followed by their conversion into inorganic dispersed particles during the processes relevant to the thermal effects on the material during its processing into compact products. This method allows to produce the composites containing 0.001 to $2 \%$ inorganic, normally oxide, fillers.

Higher filler content can be achieved by mixing components over coupling agents, i.e. substances capable of modifying the surface of filler grains or UHMWPE the way that enables a reliable bond between the materials constituting the composite $^{10-12}$.

It is organosilicon compounds containing alkoxysilyl groups that are most widely used as compounds capable of forming stable bonds with inorganic oxide particles.

One of the widely used coupling agents is 3-aminopropyltrimethoxysilane or relevant triethoxysilane. This compound was chosen to be used for modifying the surface of inorganic oxide particles added to UHMWPE-based composite material.

For example, these compounds should combine relatively low density with a high attenuation factor of passing electromagnetic radiation flux or a flow of either charged or neutral elementary particles. Oxides of the elements that have significant nuclear mass have such properties. In practice, compounds of the elements, which nuclear mass is either equal to exceeds that of the barium, such as REE, tantalum, tungsten, lead and bismuth are widely used. At the same time, such factors as material's density and its applicability for industrial composite production are also taken into account. Among the materials that have average density values, REE oxides, which have an ability attenuate electromagnetic radiation fluxes and a large neutron capture cross section, are particularly highlighted. Gadolinium and its compounds are among the most effective substances against neutron radiation. A natural mixture of gadolinium isotopes has a thermal neutron capture cross section of 49,000 barn, while that of ${ }^{157} \mathrm{Gd}$ isotope is 254,000 barn. Such characteristics make it possible to use composites containing gadolinium compounds for protection against neutron radiation. Samarium and dysprosium also refer to the elements of large thermal neutron capture cross section. The cermets based on metals and dysprosium oxide are used in nuclear reactor structures as means to control and if necessary, reduce the intensity of nuclear material's fission processes due to the absorption of thermal neutrons; other dysprosium compounds potentially applicable as thermal neutron absorbers are actively studied as well. Dysprosium titanates, zirconates and hafnates have high thermal stability and can be used in high-temperature and intense radiation field environment ${ }^{13}$. Particular significance of dysprosium as a material of protective compositions is due to the fact that the interaction between the mixture of natural dysprosium isotopes and its neutrons leads to producing isotopes of significant thermal neutron capture cross-section values.

We posed the problem of studying the possibility of introducing rare-earth element oxides into the matrix of an ultrahigh-molecular polymer up to $40 \%$ by weight and studying the effect of introducing these excipients on oxidative and thermal degradation.

\section{EXPERIMENTAL}

The process of producing composite materials based on a metal oxide and UHMWPE comprises four stages. At the first stage, metal oxides are modified with 3-aminopropyltriethoxysilane in the solvent. The second stage is sulfonating UHMWPE powder in the carbon tetrachloride medium. The third stage is mixing the metal oxide and UHMWPE produced at the first and second stages. The fourth stage is manufacturing a composite material from the powder produced at the previous stage using thermo-pressing method. 


\section{Filler particle surface modification}

The metal oxides used as fillers are normally compounds featured by poor adhesion to UHMWPE-like carbo-chain polymers. This phenomenon is due, among other things, to the presence of polar groups on the surface of oxide particles. Carbo-chain polymers do not contain polar groups capable of forming bond with the relevant groups on the surface of filler particles. Achieving the formation of stable bonds between polymer and filler particles requires changing the nature of each material's surface. A process, technically dubbed "coupling", which implies treating an inorganic material with various compounds, which form a chemical bond with groups located on the particle surface, is used for changing properties of the surface of oxide particles. Hydroxyl groups $-\mathrm{OH}$ are normally present on the surface of oxide particles.

The compounds, which readily react with hydroxyl groups, e.g. alkoxysilanes, are used for forming a chemical bond. The interaction between alkoxysilanes and surface hydroxyl groups of oxide particles leads to formation of a stable siliconoxygen bond, which, in turn, leads to substantial changes in properties of the surface of filler particles, caused by replacing hydroxyl groups with a organosilicon fragment. Depending on the type of alkoxysilane used, a variety of structural fragments can be attached to the surface of oxide particles, which have a certain impact on the affinity for the polymer matrix. Alkoxysilanes such as methyltrimethoxysilane, vinyltrimethoxysilane and 3-aminopropyltriethoxysilane were examined. Although the surface modification process was carried out in various media, it was found that the best interaction occurred in the o-xylene medium at the temperatures exceeding $100^{\circ} \mathrm{C}$. Under such conditions a layer of organosilicon compounds, which provides a substantial increase in particle hydrophobicity, thus contributing to its improved coupling with the polymer matrix, is formed on the surface of oxide particles. Of the alkoxysilanes tested, 3-aminopropyltriethoxysilane was selected, since 3-aminopromyl groups formed on the particle surface are reactive and can interact with the UHMWPE grain surface modified in a certain way, accompanied by forming stable bonds. Thus, during the studies an optimal method of modifying the surface of filler particles was determined, which implies an interaction between metal oxides and 3-aminopropyltriethoxysilane in the xylene medium at $120-144{ }^{\circ} \mathrm{C}$. It should be noted that moisture needs to be carefully removed from the solvent, otherwise hydrolytic decomposition of the alkoxysilane may occur. Moreover, the instrumentation of the process should make it possible to remove ethanol vapors formed during the interaction as a by-product from the reaction zone.

\section{Samarium oxide modification}

$23.4 \mathrm{~g}$ of samarium oxide and $80 \mathrm{ml}$ of o-xylene were fed into $250-\mathrm{cm}^{3}$ three-neck flask equipped with magnetic mixer, temperature gauge, reflux condenser and calcium chloride tube, using glass funnel. The solution was then mixed with a magnetic mixer and heated at $144{ }^{\circ} \mathrm{C}$ for 2 hours. After that, $1 \mathrm{ml}$ of 3-aminopropyltriethoxysilane dissolved in $30 \mathrm{ml}$ of o-xylene was added through the drop funnel for $20 \mathrm{~min}$. followed by resuming heating and mixing for another 2 hours. The solution was then cooled down and filtered out using «blue ribbon" filter. The powder produced was dried in the drying cabinet at $55-60^{\circ} \mathrm{C}$ for $5 \mathrm{~h}$, which led to producing $22.7 \mathrm{~g}$ of beige powder, with the product yield of $93.2 \%$.

\section{UHMWPE grain surface modification}

UHMWPE refers to carbo-chain polymers featured by poor adhesion to oxide fillers, which is due to the absence of polar groups in the UHMWPE. The chemical nature of UHMWPE is such that this material shows extremely poor interaction with various reagents accompanied by formation of certain functional fragments in the polymer chain. However, there are a number of chemical processes that allow to add functional groups to the polymer chain. The subject of particular interest is UHMWPE sulfonation, since the sulfonation in the polymer chain leads to the formation of polar sulfonic groups capable of interacting with suitable structural fragments on the surface of filler particles treated with 3-aminopropyltriethoxysilane.

Sulfurization can be carried out only by using exceptionally active sulfonating agents, which is due to poor reactivity and inertness of UHMWPE. Chlorosulfonic was used as an active sulfonating agent. It was found out that carbon tetrachloride 
was the optimal medium for such interaction. Chlorosulfonic acid solution in the carbon tetrachloride was initially added to the UHMWPE suspension in the same solvent. Given the combination of properties, it was shown that using chlorosulfonic acid was the only way achieving controlled process of modifying the UHMWPE surface not accompanied by oxidative processes, which lead to the destruction of polymer macromolecules.

$400 \mathrm{~g}$ of UHMWPE powder were fed into $2000-\mathrm{cm}^{3}$ four-neck flask equipped with magnetic mixer, temperature gauge and reflux condenser, using plastic funnel, followed by adding $700 \mathrm{ml}$ of carbon tetrachloride. The mixture was then heated at $70-75^{\circ} \mathrm{C}$, accompanied by continuous $1 \mathrm{~h}$ mixing. After that, $2 \mathrm{ml}$ of chlorosulfonic acid dissolved in $50 \mathrm{ml}$ of carbon tetrachloride were added through the drop funnel, followed by resuming heating and mixing. In $20 \mathrm{~min}$. the mixture was filtered out using ceramic filter. The powder produced was dried in the drying cabinet at $55-60{ }^{\circ} \mathrm{C}$ for $5 \mathrm{~h}$, which led to producing $396 \mathrm{~g}$ of beige powder, with the product yield of $98 \%$.

\section{Mixing oxide filler and UHMWPE to produce a composite material}

The particles of oxides and UHMWPE pre-treated with chemical reagents must be evenly mixed to achieve established filler concentration and its homogeneous distribution in the polymer matrix. The density of oxide filler particles is substantially higher than that of UHMWPE, which leads to gravitational separation of filler particles in the absence of additional forces ensuring a stable bond between filler particles and UHMWPE. Chemical surface modification contributes to the emergence of stable contacts between composite material components to a certain degree, but it is necessary to achieve dispersion and even distribution of particles to provide for required characteristics of the target composite. For this purpose, the pre-treated component particles are put into dispersion medium and subjected to external impact for establishing dispersion equilibrium. Mechanical methods and methods based on combining mechanical mixing with interlacing alternating high-frequency changes in local density and medium temperature induced by ultrasound can be used as external impact.

An organic liquid capable of effectively wetting both filler particles and polymer particles is selected as dispersion medium, while taking into account the need to bring UHMWPE grains' solvent adsorption to minimum, since thermo-pressing volatile components of the compositions may lead to the formation of local cavities, which have an adverse effect on material properties. As the study results showed, it is 2-propanol that has an optimal combination of characteristics as dispersion medium.

The ultrasonic impact on dispersions containing both UHMWPE grains and filler particles leads to local temperature and pressure maxima, which, in turn, contributes to the formation of additional functional groups on particle surfaces. Being subjected to ultrasound, particle aggregates of particles are destructed, with their total surface area increasing, which contributes to homogeneous mixing that depends on the surface area of the particles constituting the system. In technical terms the ultrasonic impact is combined with mixing to prevent gravitational separation of the particles of different density.

Once the dispersion process is over, the dispersion medium is removed, yet the mixing process does not stop. Thus, it is possible to produce a powder comprising firmly bonded filler and UHMWPE particles.

Mixing modified samarium oxide and sulphonated UHMWPE powder (samarium oxide content: $20 \%) 6.2 \mathrm{~g}$ of modified samarium oxide, $24.9 \mathrm{~g}$ of sulphonated UHMWPE powder and $100 \mathrm{ml}$ of IPA were fed into $500 \mathrm{~cm}^{3}$ rotary evaporator powder flask. The flask was then subjected to the ultrasound accompanied by simultaneous mixing for $30 \mathrm{~min}$. and vacuum-boiled down till dry. The powder produced was dried in the drying cabinet at $55-60{ }^{\circ} \mathrm{C}$ for 2 hours. 


\section{RESULTS AND DISCUSSIONS}

The composite material based on a metal oxide and UHMWPE is a powder with its particle size range of 50 to 200 microns. UHMWPE is insoluble in water and tends to swell in such solvents as carbon tetrachloride, chloroform, benzene, benzyl alcohol, xylene, decalin and higher paraffins.

Specific properties of UHMWPE, which constitutes a CM, also determine its specific applications. UHMWPE is used in cases conventional HDPE and many other thermoplastics do not withstand harsh operating conditions. In addition to their ability of attenuating electromagnetic radiation fluxes, REE oxides have large neutron capture cross section. Gadolinium and its compounds are among the most effective substances against neutron radiation ${ }^{14}$. Such characteristics allow to use a CM containing gadolinium compounds for protection against neutron radiation. Samarium and dysprosium also refer to the elements of large thermal neutron capture cross section. Particular significance of

Table 1: Heat resistance UHMWPE-based composite materials

\begin{tabular}{|c|c|c|c|c|c|c|}
\hline Metal oxide & $\begin{array}{l}\text { Determination of metal } \\
\text { oxide content, \%mass }\end{array}$ & $\begin{array}{l}\text { Density, } \\
\mathrm{kg} / \mathrm{m}^{3}\end{array}$ & $\begin{array}{l}\text { Argon } \\
T_{d 5},{ }^{\circ} \mathrm{C}\end{array}$ & $\begin{array}{l}\text { Argon } \\
\mathrm{T}_{\mathrm{d} 10},{ }^{\circ} \mathrm{C}\end{array}$ & $\mathrm{T}_{\mathrm{d} 5}{ }^{\mathrm{Air}}{ }^{\circ} \mathrm{C}$ & $\mathrm{T}_{\mathrm{d} 10}{ }^{\mathrm{Air}}{ }^{\circ} \mathrm{C}$ \\
\hline \multirow{3}{*}{$\begin{array}{l}\text { without metal } \\
\text { oxide (pure } \\
\text { UHMWPE) }\end{array}$} & & & & & & \\
\hline & 0,0 & 0,962 & 434 & 448 & 368 & 392 \\
\hline & 10,0 & 1,020 & 455 & 463 & 312 & 358 \\
\hline \multirow[t]{2}{*}{$\mathrm{WO}_{3}$} & 20,0 & 1,011 & 452 & 461 & 328 & 388 \\
\hline & 39,2 & 1,149 & 456 & 466 & 300 & 336 \\
\hline \multirow{4}{*}{$\mathrm{Dy}_{2} \mathrm{O}_{3}$} & 10,0 & 0,985 & 451 & 462 & 348 & 397 \\
\hline & 19,9 & 0,961 & 451 & 461 & 334 & 391 \\
\hline & 39,8 & 1,208 & 452 & 464 & 396 & 407 \\
\hline & 10,0 & 0,966 & 451 & 462 & 318 & 342 \\
\hline \multirow{3}{*}{$\mathrm{Gd}_{2} \mathrm{O}_{3}$} & 18,8 & 0,980 & 444 & 459 & 342 & 397 \\
\hline & 37,4 & 1,065 & 443 & 458 & 360 & 402 \\
\hline & 10,0 & 1,015 & 457 & 468 & 389 & 401 \\
\hline \multirow[t]{2}{*}{$\mathrm{Sm}_{2} \mathrm{O}_{3}$} & 19,4 & 1,067 & 449 & 464 & 345 & 393 \\
\hline & 38,7 & 1,186 & 456 & 471 & 345 & 412 \\
\hline
\end{tabular}

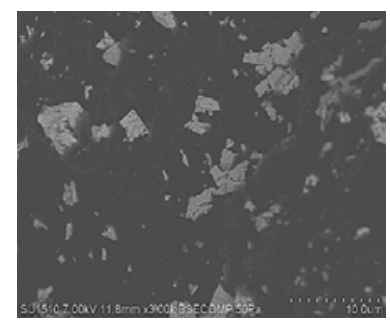

(a)

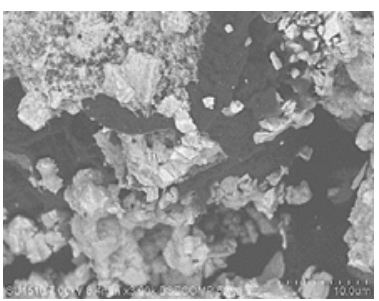

(C)

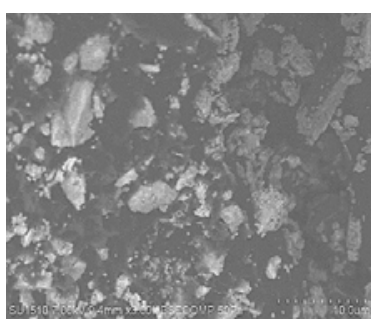

(b)

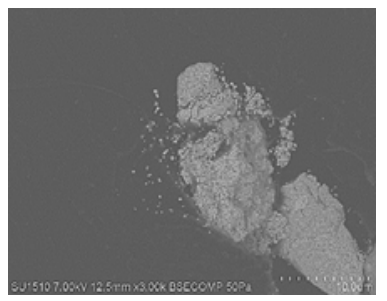

(d)

Fig. 1. SEM image of the composite structure: (a) - UHMWPE+ $20 \% \mathrm{Sm}_{2} \mathrm{O}_{3}$, (b) - UHMWPE $+20 \% \mathrm{Gd}_{2} \mathrm{O}_{3}$, (c) - UHMWPE + 20\% $\mathrm{Dy}_{2} \mathrm{O}_{3}$, (d) - UHMWPE $+20 \% \mathrm{WO}_{3}$ 
dysprosium as a material of protective compositions is due to the fact that the interaction between the mixture of natural dysprosium isotopes and its neutrons leads to producing isotopes of significant thermal neutron capture cross-section values ${ }^{13}$.

All UHMWPE-based composite materials were examined using atomic emission spectrometry and inductively coupled plasma to determine the gadolinium oxide $\left(\mathrm{Gd}_{2} \mathrm{O}_{3}\right)$, dysprosium oxide $\left(\mathrm{Dy}_{2} \mathrm{O}_{3}\right)$, tungsten oxide $\left(\mathrm{W}_{2} \mathrm{O}_{3}\right)$ and samarium oxide $\left(\mathrm{Sm}_{2} \mathrm{O}_{3}\right)$ contents. The thermal resistance of all the samples obtained was tested as well.

The data obtained show an increased thermal stability with increasing filler content. The introduction of tungsten oxide, however, leads to a decrease in oxidation resistance, while oxides of dysprosium, gadolinium, and samarium with the contain more than $20 \%$ do not lead to significant oxidative degradation.

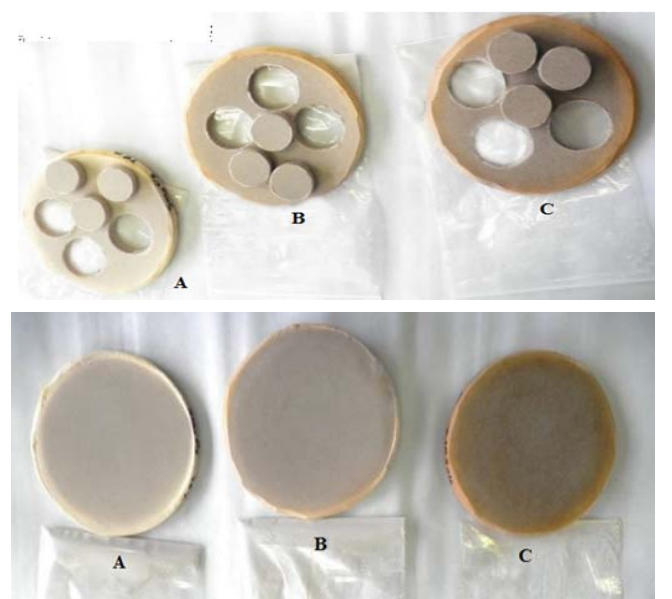

A - UHMWPE $+40 \%$ mass $\mathrm{Sm}_{2} \mathrm{O}_{3}$; B- UHMWPE $+20 \%$ mass $\mathrm{Sm}_{2} \mathrm{O}_{3} ; \mathrm{C}-\mathrm{UHMWPE}+10 \%$ mass $\mathrm{Sm}_{2} \mathrm{O}_{3}$

Fig. 2. Samples of composite materials based on metal oxide-containing UHMWPE
The molding materials produced were loaded into the mold and subjected to pressing by means of $250 \times 250-\mathrm{mm}$ curing heat press machine at $200^{\circ} \mathrm{C}$ and $10 \mathrm{MPa}$ for $20 \mathrm{~min}$. followed by cooling down at the rate of $3-4{ }^{\circ} \mathrm{C} /$ minutes.

\section{CONCLUSION}

This developed method of producing composite materials based on UHMWPE and metal oxides such as REE oxides, including gadolinium, dysprosium and tungsten oxides, allows to produce composites with its filler content of up to $40 \%$. The obtained TGA data showed that, with a filler content more than $30 \%$, the thermal stability is maintained. This method is based on modifying oxide filler surface using organosilicon coupling agents in the acid catalyst environment. All resulted composite materials based on UHMWPE and gadolinium oxide $\left(\mathrm{Gd}_{2} \mathrm{O}_{3}\right)$, dysprosium oxide $\left(\mathrm{Dy}_{2} \mathrm{O}_{3}\right)$, tungsten oxide $\left(\mathrm{W}_{2} \mathrm{O}_{3}\right)$ and samarium oxide $\left(\mathrm{Sm}_{2} \mathrm{O}_{3}\right)$ have increased thermal resistance with increasing filler content up to $40 \%$. The oxides of dysprosium, gadolinium, and samarium with the contain more than $20 \%$ do not lead to oxidative degradation.

\section{ACKNOWLEDGEMENT}

Applied researches are carried out with state financial support represented by the Ministry of Education of Russia under the Agreement on granting subsidies No14.625.21.0035 of October 27, 2015. (Unique identifier of Applied Scientific Researches (project) RFMEFI62515X0035).

\section{REFERENCES}

1. Mikitaev A. K.; Kozlov G.V.; Yanovsky Yu.G.; Description of the mechanical properties of dispersed-filled nanostructured polymeric composites within the framework of fractal analysis. Phys. mesomech., 2014, 17(6), 71-79.

2. Kozlov G.V. Structure and properties of particulate-filled polymer nanocomposites.
Phys. Usp., 2015, 58(1), 33-60.

3. Supova M; Martynkova G.S.; Barabaszova K. Effect of Nanofillers Dispersion in Polymer Matrices. Sci. Adv. Mater., 2011, 3(1), 1-25.

4. Mittal V. Modeling and Prediction of Polymer Nanocomposite Properties. Weinheim: Wiley-VCH., 2013. 
5. Andrievsky R.A. Nanomaterials: the concept and modern problems. Russian Chemical Journal., 2002, 46(5), 50-56.

6. Lomakin S. M., Zaikov G. E.; Polymeric nanocomposites of low combustibility based on layered silicates. High-Molecular Compounds., 2005, 47(1), 104-120.

7. Bobryshev A. N.; Synergetics of composite materials. Lipetsk: NGO ORIUS., 1994.

8. Bartenev G.M.; Zelenev Yu.V.; Physics and mechanics of polymers. Moscow: Higher School., 1983.

9. Tugov I. I.; Shaulov A.Yu. The modulus of elasticity of dispersed-filled composites. Highmolecular. Cpd. B., 1990, 32(7), 527-529.

10. Bashorov M. T.; Kozlov G. V.; Mikitaev A. K. Nanostructures and properties of amorphous glassy polymers. Moscow:
Publishing House of the Russian Chemical Technical University. DI. Mendeleev., 2010.

11. Magomedov G.M.; Kozlov G.V.; Zaikov G.E. StructureandProperties of Cross-LinkedPolymers. Shawbury: Smithers Group Co., 2011.

12. Zhu Y.; Li H., Koltypin Y.; Hacohen Y.R.; Gedanken A. Sonochemical synthesis of titania whiskers and nanotubes. Chemical Communications., 2001, 24, 2616-2617

13. Amit S.; Sharma B.P. Development of Dysprosium Titanate Based Ceramics. Journal of the American Ceramic Society., 2005, 88(4), 1064-1066.

14. Hu, H., Wang, Q., Qin, J., Wu, Y., Zhang, T., Xie, Z. Study on Composite Material for Shielding Mixed Neutron and gamma-rays. IEEE Transactions on Nuclear Science., 2008, 55(4), 2376-2384. 